\title{
Bundesverfassungsgericht und Klimaschutz - wenn die Zukunft über die Gegenwart mitentscheiden darf
}

\author{
Katharina Kotulla und Michael Kotulla
}

(c) Der/die Autor(en) 2022. Dieser Artikel ist eine Open-Access-Publikation.

Das Bundesverfassungsgericht (BVerfG) hat in seiner weithin beachteten und für viele überraschenden Entscheidung vom 24.3. 2021 nicht nur dem Klimaschutz zu einem verfassungsrechtlich neuen Stellenwert verholfen, sondern auch in den Grundrechten einen bisher nicht gekannten intertemporalen Charakter identifiziert. Der nachfolgende Beitrag befasst sich kritisch mit der daraus resultierenden Bedeutung und den damit verbundenen Folgen des Judikats.

\section{Einführung}

Hintergrund der ohne mündliche Verhandlung und einstimmig ergangenen Entscheidung des $\mathrm{BVerfG}^{1}$ sind vier sich gegen Vorschriften des Bundes-Klimaschutzgesetzes (KSG) vom 12.12.2019² und die Unterlassung zusätzlicher staatlicher Maßnahmen zur Reduzierung von Treibhausgasemissionen gerichtete Verfassungsbeschwerden, darunter drei auch von Minderjährigen erhoben. Insoweit wird insbesondere moniert, der Staat habe keine ausreichenden Regelungen zur alsbaldigen Reduzierung von Treibhausgasen, allem voran von Kohlendioxid $\left(\mathrm{CO}_{2}\right)$, geschaffen. Diese aber seien erforderlich, um die Erwärmung der Erde bei 1,5 Grad Celsius oder wenigstens deutlich unter 2 Grad Celsius zu begrenzen. Im Kern legt das Gericht dabei als Gegenstand seiner Prüfung den $₫ 3$ Abs. 1 S. 2 sowie den $\int 4$ Abs. 1 Satz 3 (i.V.m. Anlage 2) und Abs. 6 KSG zugrunde, deren Vereinbarkeit es vornehmlich mit dem aus Art. 2 Abs. 1 GG hergeleiteten Schutz,,sämtlicher menschlicher Freiheitsbetätigungen" ${ }^{3}$ und dem in Art. 2 Abs. 2 S. 1 GG verankerten Grundrecht auf Gesundheit und Leben der Beschwerdeführer (Bf.) prüft. Bemerkenswerterweise geht das Gericht insoweit von einer ,objektivrechtlichen Schutzverpflichtung“ des Staates ,,auch in Bezug auf künftige Generationen“ aus. ${ }^{4}$ Ergänzend hierzu wird als zentraler Maßstab der aus Art. 20a GG abgeleitete Auftrag des Staates zum Schutz des Klimas heranzugezogen. Dabei hatten die Verfassungsbeschwerden überhaupt nur insoweit Erfolg, als $₫ 3$ Abs. 1 S. 2 und $\$ 4$ Abs. 1 Satz 3 (i. V.m. Anlage 2) KSG als mit den Grundrechten für unvereinbar befunden wurden, soweit diese lediglich jährliche $\mathrm{CO}_{2}$-Emissionsreduzierungsmengen bis zum Jahre 2030 vorsehenden Regelungen keine hinreichenden Maßgaben für die weitere Emissionsreduzierung ab dem Jahre 2031 enthielten.

Die Reaktionen auf diesen Beschluss sind gerade in der rechtswissenschaftlichen Literatur bis jetzt nicht nur in ihrer Anzahl, sondern auch in ihrer Bewertung beachtlich. Sie fallen zwar überwiegend auffällig positiv ${ }^{5}$, wenn auch

Cand. jur. Katharina Kotulla,

Universität Bielefeld,

Bielefeld, Deutschland

Prof. Dr. Michael Kotulla, M. A.,

Lehrstuhl für Öffentliches Recht, insbesondere Umweltrecht,

und Verfassungsgeschichte, Geschäftsführender Direktor

des Instituts für Umweltrecht, Universität Bielefeld,

Bielefeld Deutschland durchaus mitunter noch unzufrieden, weil weitere Fragen aufwerfend ${ }^{6}$ bzw. nicht weitgehend genug ${ }^{7}$ oder doch eher unkritisch ${ }^{8}$, wenn nicht sogar euphorisch aus, sofern der Entscheidung etwa die Bedeutung einer ,revolutionären Wende" in prozessualer wie materieller Hinsicht bescheinigt wird, da nunmehr gerichtlich nachgeprüft werden könne, ob der Gesetzgeber seiner Pflicht zum Klimaschutz effektiv nachkomme. ${ }^{9}$ Dennoch finden sich mitunter auch kritische Stimmen, die den Beschluss für ,ökonomisch und ökologisch unsinnig, verfassungsrechtlich falsch“ halten. ${ }^{10}$ Dazwischen angesiedelt gibt es vereinzelt Bedenken über grundrechtsdogmatisch bedeutsame Aussagen des Gerichts ${ }^{11}$ und Ansätze zu deren Übertragbarkeit etwa auf andere Umweltbereiche ${ }^{12}$ sowie Überlegungen zur Geltung der deutschen Grundrechte über die nationalen Grenzen hinaus ${ }^{13}$. Schließlich gibt es verhalten skeptische Reaktionen, die etwa bezweifeln, ob es überhaupt die Aufgabe des BVerfG sein könne, eigentlich dem demokratischen legitimierten Gesetzgeber zukommende Rahmenentscheidungen gleichsam an sich zu ziehen. ${ }^{14}$

\section{Staatsziel ,Klimaschutz“6}

Das BVerfG stellt - übrigens nicht zum ersten $\mathrm{Mal}^{15}$ - fest, dass Art. 20a GG den Staat zum Klimaschutz verpflichte. ${ }^{16}$ Hinter dieser groben Verkürzung versteckt sich die Verpflichtung des Staates zum Schutz vor den nachteiligen

1) BVerfG, Beschl. v. 24.3.2021 - 1 BvR 2656/18, 1 BvR 78/20, 1 BvR 96/20, 1 BvR 288/20.

2) BGBl. I S. 2513

3) BVerfG, Beschl. v. 24.3.2021 - 1 BvR 2656/18, 1 BvR 78/20, 1 BvR 96/20, 1 BvR 288/20, Rdnr. 184.

4) BVerfG, Beschl. v. 24.3.2021 - 1 BvR 2656/18, 1 BvR 78/20, 1 BvR 96/20, 1 BvR 288/20, LS 1.

5) Vgl. etwa Schlacke, NVwZ 2021, 912, 914; Wagner, NJW 2021 , $2256 \mathrm{ff}$.

6) Wickel, ZUR 2021, $332 \mathrm{ff}$; Frenz, EnWZ 2021, $201 \mathrm{ff}$.; Burgi, NVwZ 2021, $1401 \mathrm{ff}$.

7) Ekardt/Heß, ZUR 2021, $579 \mathrm{ff}$.

8) Breidenbach, ZRP 2021, $244 \mathrm{ff}$.

9) Callies, ZUR 2021, 335, 335.

10) Murswiek, in: Welt v. 29.8.2021, Stand 12.12.2021, abrufbar unter https://www.welt.de/wirtschaft/plus233215175/KlimaUrteil-Oekonomisch-und-oekologisch-unsinnig-verfassungsrechtlich-falsch.html.

11) So etwa: Faßbender, NJW 2021, 2085, $2088 \mathrm{ff}$; E. Hofmann, NVwZ 2021, 1587 ff.; Schlacke, NVwZ 2021, $912 \mathrm{ff}$.

12) Stubenrauch, ZUR 2021, $617 \mathrm{ff}$.

13) Markus, ZUR 2021, $595 \mathrm{ff}$.

14) Ruttloff/Freihoff, NVwZ 2021, 917 ff.; Frenz, EnWZ 2021, 201, 208.

15) Vgl. BVerfG, Beschl. v. 13.3.2007-1 BvF 1/05, BVerfGE 118 , 79, 110 f.; Urt. v. 5.11.2014 - 1 BvF 3/11, BVerfGE 137, 350, 368f.u. 378; Beschl. v. 30.6.2020 - 1 BvR 1679/17, 2190/17, BVerfGE 155, 238, 278.

16) BVerfG, Beschl. v. 24.3.2021 - 1 BvR 2656/18, 1 BvR 78/20, 1 BvR 96/20, 1 BvR 288/20, Rdnr. $197 \mathrm{f}$. 
Folgen des vom Menschen verursachten Klimawandels. Diese wird offenbar aus dem dort als Staatsziel verankerten Schutzgebot für die ,natürlichen Lebensgrundlagen“ hergeleitet, ${ }^{17}$ als deren Bestandteil auch das ,Klima“ zu gelten hat. Doch bleibt im Weiteren die inhaltliche Umschreibung dessen, was den Schutzgegenstand und damit den KlimaBegriff ausmachen soll, bestenfalls unklar. Jedenfalls lässt sich das Gericht insoweit auf keine wirklichen Definitionen des von Art. 20a GG erfassten Klimas und dessen Wandel ein. Beide werden wohl als allgemein bekannt vorausgesetzt. Anders aber als die in Art. 20a GG ausdrücklich erwähnten ,natürlichen Lebensgrundlagen“ und „Tiere“ handelt es sich bei dem Klimaschutzbegriff indes keineswegs um einen eindeutig aus sich selbst heraus bestimmbaren Terminus.

Zumindest in der Meteorologie umfasst Klima üblicherweise die Gesamtheit der physikalischen Vorgänge, die den mittleren Zustand der Atmosphäre an irgendeiner Stelle der Erdoberfläche kennzeichnen, außerdem die Gesamtheit der Witterungen (Wetterabläufe) eines längeren oder kürzeren Zeitabschnitts, wie sie durchschnittlich in diesem Zeitraum einzutreten pflegen. ${ }^{18}$ Stattdessen wird vom BVerfG mit der „,mittlere(n) Temperatur der Erde“ lediglich eine „Zentrale Leitgröße für den klimatischen Zustand des Erdsystems insgesamt " bemüht; ${ }^{19}$ weswegen hier das eigentlich begriffsinhaltlich deutlich gehaltvollere Klimaschutzgebot im Kern auf die bloße Einhaltung einer Temperaturschwelle heruntergezont ist, bei der die als Folge von in die Erdatmosphäre gelangenden anthropogenen Treibhausgasemissionen verursachte Erwärmung der Erde angehalten werden solle. ${ }^{20}$ Dabei sind mit den Treibhausgasemissionen hier letztlich ausschließlich $\mathrm{CO}_{2}$-Emissionen gemeint. Dies erscheint schon deshalb unbefriedigend, da auf zahlreiche andere für die Erderwärmung mitursächlichen Treibhausgase, wie etwa Methan $\left(\mathrm{CH}_{4}\right)$ oder Lachgas $\left(\mathrm{N}_{2} \mathrm{O}\right)^{21}$, überhaupt nicht eingegangen wird. Auch zu sonstigen möglichen Erderwärmungsfaktoren - wie etwa den global festzustellenden großflächigen Verlust von Waldbeständen - findet sich kein Wort. Überhaupt scheint das Gericht in seiner Argumentation von den anthropogen verursachten $\mathrm{CO}_{2}$-Emissionen als der mit Abstand wesentlichsten Ursache für die derzeitige Erderwärmung auszugehen. Die Richtigkeit der Annahme dürfte in dieser Verengung gewiss anzuzweifeln sein. Die grundsätzliche Notwendigkeit einer Reduzierung anthropogener $\mathrm{CO}_{2}$-Emissionen als eine $\mathrm{Maß-}$ nahme gegen die Erderwärmung mag durchaus sinnvoll, doch ohne entsprechend flankierte weitere wirkungsvolle Schutzmaßnahmen wohl kaum ausreichend sein. Kurzum: die einseitige Ausrichtung des vom BVerfG für notwendig erachteten Klimaschutzes auf die $\mathrm{CO}_{2}$-Reduzierung greift viel zu kurz. Dies gilt übrigens ungeachtet der vom $\mathrm{Ge}-$ richt seiner eigenen Einschätzung zugrunde gelegten wissenschaftsbasierten Arbeiten politikberatender Expertenräte wie dem Weltklimarat (Intergovernmental Panel on Climate Change - IPCC) und dem Sachverständigenrat für Umweltfragen (SRU).

Die Steuerungswirkung des Art. 20a GG selbst bleibt angesichts der dort allenfalls vage ableitbaren klimaschutzrelevanten Inhalte aber eher gering. Dennoch sieht das Gericht in der Erderwärmung und deren Begrenzung ein globales Phänomen, das nicht durch die Klimaschutzbeiträge eines Staates allein gelöst werden könne. Dementsprechend habe der Klimaschutzauftrag des Art. 20a GG ,von vornherein eine besondere internationale Dimension“; 22 weswegen sich für den deutschen Staat immerhin die Verpflichtung ergebe, eine Lösung des Klimaschutzproblems gerade auch auf überstaatlicher Ebene zu suchen. Erst derart völkerrechtlich eingebettet könnten nationale Maßnahmen die von Art. 20a GG geforderte Wirkung entfalten, auch wenn sie für sich genommen das Klimaproblem nicht zu lösen vermöchten. ${ }^{23}$ Ungeachtet dessen verpflichte Art. 20a GG selbst dann zu nationalen Klima- schutzanstrengungen, wenn eine internationale Kooperation nicht zustande käme. ${ }^{24}$ Zwar falle der bundesdeutsche Anteil an den weltweiten $\mathrm{CO}_{2}$-Emissionen mit 2\% für sich genommen eher gering aus, doch seien Deutschlands Klimaschutzbemühungen als Teil einer weltweiten Gesamtanstrengung geeignet, das Ende des Klimawandels herbeizuführen. ${ }^{25}$ Vor diesem Hintergrund erscheint die Feststellung des Gerichts, der Gesetzgeber habe mit der Statuierung einer der dem Art. 2 Abs. 1 lit. a) des - am 28. 9. 2016 von der Bundesrepublik Deutschland ratifizierten - Pariser Klima-Übereinkommens vom 12.12.2015 26 entsprechenden Temperaturschwelle die grundlegende Ausrichtung des nationalen Klimaschutzrechts gerade in der Weise bestimmt, die dem deutschen Staat die Möglichkeit eröffne, seinen verfassungsrechtlichen Auftrag zum Klimaschutz international eingebettet durch eigene Anstrengungen effektiv zu erfüllen, ${ }^{27}$ zumindest folgerichtig. Das BVerfG versteht denn auch das dort festgesetzte Ziel, den Anstieg der durchschnittlichen Erdtemperatur deutlich unter 2 Grad Celsius über dem vorindustriellen Niveau zu halten und Anstrengungen zu unternehmen, um den Temperaturanstieg möglichst auf 1,5 Grad Celsius über dem vorindustriellen Niveau zu begrenzen (,Paris-Ziel““), als die den Art. 20a GG konkretisierende Basis des deutschen Klimaschutzrechts, die auch in $₫ 1 \mathrm{~S} .3$ des deutschen KSG als eine mögliche zulässige Konkretisierung des Klimaschutzgebotes zugrunde gelegt wird. ${ }^{28} \mathrm{Zwar}$ ist das in $\$ 1$ S. 3 KSG gesetzte, auf $\mathrm{CO}_{2}$-Reduzierung beschränkte Temperaturbegrenzungsziel gemessen an der mit Art. 20a GG vorgegebenen Klimaschutzaufgabe verfassungsrechtlich wohl schwerlich zu beanstanden. Doch könnten - so das Gericht weiter - stattdessen grundsätzlich auch andere geeignete Maßnahmen getroffen bzw. Ziele im Rahmen des gebotenen Klimaschutzes festgelegt werden. Wenn sodann wiederum an anderer Stelle der ,strikten Wahrung des durch Art. 20a GG vorgegebenen Emissionsrahmens“ (gemeint ist die Erreichung des „Paris-Zieles“) das Wort geredet wird und das „Paris-Ziel“" sogar eine ,verfassungsrechtliche Maßgabe“ sein solle, ${ }^{29}$ steht dies krass im Widerspruch dazu. Denn für sich betrachtet gibt Art. 20a GG we-

17) BVerfG, Beschl. v. 24.3.2021 - 1 BvR 2656/18, 1 BvR 78/20, 1 BvR 96/20, 1 BvR 288/20, vgl. Rdnr. 200.

18) In diesem Sinne etwa Brockhaus Enzyklopädie in 24 Bänden, Bd. 12 (Kir - Lag), 19. Aufl. 1990, Art. „Klima“, S. 83.

19) BVerfG, Beschl. v. 24.3.2021 - 1 BvR 2656/18, 1 BvR 78/20, 1 BvR 96/20, 1 BvR 288/20, Rdnr. 198.

20) BVerfG, Beschl. v. 24.3.2021 - 1 BvR 2656/18, 1 BvR 78/20, 1 BvR 96/20, 1 BvR 288/20, Rdnr. 198.

21) Siehe dazu bereits: v. Witzke/Noleppa, Methan und Lachgas Die vergessenen Klimagase, in: WWF Deutschland (Hrsg.), Frankfurt/M., Stand: November 2007; Bayerisches Landesamt für Umwelt 2020: „Lachgas“, Stand 12.12.2021, abrufbar unter https://www.lfu.bayern.de/klima/klimaschutz/treibhausgase/lachgas/index.htm; vgl. auch: EU-Methan-Strategie v. 14.10.2020, COM(2020) 663 final; COP 26, The Global Methane Pledge, Stand 12.12.2021, abrufbar unter https://ukcop26. org/wp-content/uploads/2021/11/COP26-Presidency-OutcomesThe-Climate-Pact.pdf, S. 15.

22) BVerfG, Beschl. v. 24.3.2021 - 1 BvR 2656/18, 1 BvR 78/20, 1 BvR 96/20, 1 BvR 288/20, Rdnr. 199.

23) BVerfG, Beschl. v. 24.3.2021 - 1 BvR 2656/18, 1 BvR 78/20, 1 BvR 96/20, 1 BvR 288/20, Rdnr. 201.

24) BVerfG, Beschl. v. 24.3.2021 - 1 BvR 2656/18, 1 BvR 78/20, 1 BvR 96/20, 1 BvR 288/20, Rdnr. 201.

25) BVerfG, Beschl. v. 24.3.2021 - 1 BvR 2656/18, 1 BvR 78/20, 1 BvR 96/20, 1 BvR 288/20, Rdnr. 202 f.

26) Siehe BGB1. II 2016, S. 1082.

27) BVerfG, Beschl. v. 24.3.2021 - 1 BvR 2656/18, 1 BvR 78/20, 1 BvR 96/20, 1 BvR 288/20, Rdnr. 210.

28) BVerfG, Beschl. v. 24.3.2021 - 1 BvR 2656/18, 1 BvR 78/20, 1 BvR 96/20, 1 BvR 288/20, Rdnr. 210.

29) BVerfG, Beschl. v. 24.3.2021 - 1 BvR 2656/18, 1 BvR 78/20, 1 BvR 96/20, 1 BvR 288/20, Rdnr. 246 
der ein bestimmtes Temperaturziel noch einen bestimmten Emissionsrahmen vor. Dies bestätigt letztlich auch das Gericht selbst, wenn es an anderer Stelle auf den im Rahmen des Art. 20a GG vorhandenen Konkretisierungsspielraum des Gesetzgebers verweist, der derzeit mit dem in $\$ 1$ S. 3 KSG gesetzten Temperaturziel gewahrt bleibe. ${ }^{30}$ Besagter Spielraum werde nur dadurch eingeschränkt, dass der Staat nicht untätig bleiben oder gänzlich ungeeignete Maßnahmen treffen dürfe. ${ }^{31}$

Das BVerfG sieht $₫ 1$ S. 3 KSG mit der dort zugrunde gelegten Temperaturmaßgabe als ,verfassungsrechtlich notwendige, grundlegende Konkretisierung des Art. 20a GG“" und weist dieser Maßgabe ,verfassungsrechtliche Orientierungsfunktion“ zu. ${ }^{32}$ Dennoch könne - was sich ohnehin von selbst versteht - mittels eines Parlamentsgesetzes die maßgebliche Klimaschutzzielsetzung und damit der verfassungsrechtliche Klimaschutzauftrag inhaltlich jederzeit geändert werden. ${ }^{33}$

Insoweit stünden - dem Gericht zufolge - jedenfalls die jährliche $\mathrm{CO}_{2}$-Emissionsreduzierungsmengen bis zum Jahre 2030 vorsehenden Regelungen in $\$ 3$ Abs. 1 S. 2 und \4 Abs. 1 S. 3 KSG i. V.m. Anlage 2 im Einklang mit dem Klimaschutzgebot des Art. 20a GG. ${ }^{34}$

\section{3. $\mathrm{CO}_{2}$-Restbudget als Maßstab für staatliche Klimaschutzmaßnahmen}

Grundsätzlich verweist das BVerfG in seiner Entscheidung auf ein der Bundesrepublik Deutschland verbleibendes nationales $\mathrm{CO}_{2}$-Restbudget. ${ }^{35}$ Es bezieht sich hierbei auf Annahmen des IPCC, die vom SRU anteilig für Deutschland übernommen worden sind und für den Zeitraum ab 2020 ein noch verbleibendes konkretes Budget i.H.v. 6,7 Gigatonnen an maximal emittierbarem $\mathrm{CO}_{2}$ vorsehen. ${ }^{36}$ Wie wenig eindeutig oder gar gesichert die vom Gericht zugrundegelegte Restbudget-Menge derzeit tatsächlich ist, zeigen etwa die wissenschaftlichen Angaben der „Helmholtz Klima-Initiative“, in denen für Deutschland ab 2021 immerhin von einem noch verbleibenden Budget von ca. 7,8 Gigatonnen $\mathrm{CO}_{2}$ ausgegangen wird ${ }^{37}$, während es aber auch deutlich niedriger ausfallende Restbudget-Schätzungen für Deutschland ab 2020 i. H.v. lediglich ca. 4,4 Gigatonnen $\mathrm{CO}_{2}$ gibt $^{38}$. Überdies gilt es als eine Schwachstelle des vom BVerfG verwendeten Restbudget-Ansatzes, dass es Sektoren - wie etwa die vorrangig andere Gase emittierende Landwirtschaft - gibt, deren klimarelevanter (Schutz-)Beitrag mit einem $\mathrm{CO}_{2}$-Budget überhaupt nicht angemessen bewertbar ist. ${ }^{39}$ Selbst das BVerfG bezeichnet die von ihm verwendeten Mengenangaben als ,,nicht zahlengenau beziffert" ${ }^{* 0}$ und kommt am Ende seiner dazu gemachten Ausführungen zu der Erkenntnis, dass es angesichts der ,derzeit“" bestehenden ,Unsicherheit bezüglich der Größe des globalen $\mathrm{CO}_{2}$-Restbudgets" ebenso zu Unsicherheiten hinsichtlich des Umfangs des nationalen Restbudgets komme. ${ }^{41}$

Zwar hat das Gericht dann an anderer Stelle wenig Hemmungen, dieses vage Restbudget als wesentlichen Bestandteil seiner Entscheidung hervorzuheben, wenn es ausführt, ein ,,auf Deutschland entfallender Anteil an (nach 2030, sc.) verbleibenden Emissionsmöglichkeiten wird ... ungeachtet der genauen Größe des Restbudgets jedenfalls zu einem nicht unerheblichen Teil verbraucht" sein, sodass ,allenfalls noch ein minimaler Rest an $\left(\mathrm{CO}_{2}-\right)$ Emissionsmöglichkeiten“ bestünde, ,der angesichts der für $2031 \mathrm{ff}$. noch zu erwartenden Emissionsniveaus kaum für ein weiteres Jahr genügte" ${ }^{42}$. Doch scheut es wohl um der damit möglicherweise verbundenen Folgen willen, diese verbleibende marginale $\mathrm{CO}_{2}$-Restbudgetvariante dem Gesetzgeber als verbindlich zu erfüllenden Rahmen vorzugeben. Bliebe diesem dann doch kein Spielraum mehr zur Regelung eines geordneten Übergangs in die immerhin erst fast zwei Jahrzehnte später, also bis zum Jahr 2050, zu er- reichende „Klimaneutralität“ (des $\mathrm{CO}_{2}$-Ausstoßes). Konsequenterweise müsste nämlich diesenfalls entweder binnen eines Jahres die Klimaneutralität herbeigeführt oder die in $\$ 3$ Abs. 1 S. 2 und $\$ 4$ Abs. 1 S. 3 KSG i.V.m. Anlage 2 erfolgten jährlichen Zwischenzielfestlegungen für Emissionsreduzierungsmengen bis zum Jahre 2030 drastisch mit unabsehbaren nachteiligen Folgen für das gesellschaftliche Leben und die Wirtschaft verschärft werden. Wohl nicht zuletzt um derartiges zu vermeiden, billigt das Gericht dem Gesetzgeber dann doch zu, ,Minderungsziele zu formulieren, ohne dabei von Beginn an eine Vorstellung davon zu entwickeln, welche Gesamtemissionsmenge noch zur Verfügung steht ${ }^{\text {"43 }}$. Erst die dadurch vorgenommene Entkoppelung des Restbudgets von den zu treffenden Maßnahmen ermöglichen es dem Gericht zufolge der Legislative, verfassungskonform nicht nur - wie in $\$ 3$ Abs. 1 S. 2 und \4 Abs. 1 S. 3 KSG i. V.m. Anlage 2 vorgesehen - für die Jahre bis 2030, sondern auch für die Jahre danach jährliche Emissionsreduzierungsmengen festzusetzen.

Dies alles ist indes vor dem Hintergrund zu sehen, dass das vom BVerfG bemühte $\mathrm{CO}_{2}$-Restbudget überhaupt keine Verankerung im Verfassungsrecht findet und insoweit auch kein verfassungsrechtlich bindender Parameter sein kann. Angesichts dessen können die vom Restbudget abgeleiteten $\mathrm{CO}_{2}$-Mengen wohl allenfalls eine vage Orientierungshilfe, keineswegs aber der (verfassungs)rechtlich verbindliche Maßstab für weitere zu treffende Maßnahmen darstellen.

\section{Grundrechte}

\subsection{Beschwerdebefugnis}

Zunächst schließt das BVerfG im Rahmen der von ihm geprüften Beschwerdebefugnis eine von den Bf. wegen der aus ihrer Sicht vom KSG zu großzügig zugelassenen Treibhausgas-Jahresemissionsmengen behauptete mögliche Ver-

30) BVerfG, Beschl. v. 24.3.2021 - 1 BvR 2656/18, 1 BvR 78/20, 1 BvR 96/20, 1 BvR 288/20, Rdnr. 211.

31) BVerfG, Beschl. v. 24.3.2021 - $1 \mathrm{BvR}$ 2656/18, $1 \mathrm{BvR}$ 78/20, 1 BvR 96/20, 1 BvR 288/20, Rdnr. 210.

32) BVerfG, Beschl. v. 24.3.2021 - 1 BvR 2656/18, 1 BvR 78/20, 1 BvR 96/20, 1 BvR 288/20, Rdnr. 213.

33) BVerfG, Beschl. v. 24.3.2021 - 1 BvR 2656/18, 1 BvR 78/20, 1 BvR 96/20, 1 BvR 288/20, Rdnr. 213.

34) BVerfG, Beschl. v. 24.3.2021 - 1 BvR 2656/18, 1 BvR 78/20, 1 BvR 96/20, 1 BvR 288/20, Rdnr. 214.

35) BVerfG, Beschl. v. 24.3.2021 - 1 BvR 2656/18, 1 BvR 78/20, 1 BvR 96/20, 1 BvR 288/20, Rdnr. 219-235.

36) BVerfG, Beschl. v. 24.3.2021 - 1 BvR 2656/18, 1 BvR 78/20, 1 BvR 96/20, 1 BvR 288/20, Rdnr. 219.

37) Siehe Werner, Unser Kohlenstoffbudget schrumpft, Stand 12.12 2021, abrufbar unter https://www.helmholtz-klima.de/aktuelles/ unser-kohlenstoff budget-schrumpft.

38) Vgl. etwa Gero Rueter, Klimaschutz: Deutschland bis 2038 klimaneutral?, Stand 12.12.2021, abrufbar unter https://www. dw.com/de/deutschland-bis-2038-klimaneutral-klimawandelexperten-der-bundesregierung-empfehlen-co2-budget-sru/a-534 27340.

39) So SRU, Umweltgutachten 2020: Für eine entschlossene Umweltpolitik in Deutschland und Europa, 2020, 2. Kap. Pariser Klimaziele, Stand 12.12.2021, abrufbar unter https://www. umweltrat.de/SharedDocs/Downloads/DE/01_Umweltgutachten/2016_2020/2020_Umweltgutachten_Kap_02_Pariser_Klimaziele.pdf?

40) BVerfG, Beschl. v. 24.3.2021 - 1 BvR 2656/18, 1 BvR 78/20, 1 BvR 96/20, 1 BvR 288/20, Rdnr. 247.

41) BVerfG, Beschl. v. 24.3.2021 - 1 BvR 2656/18, 1 BvR 78/20, 1 BvR 96/20, 1 BvR 288/20, Rdnr. 247.

42) BVerfG, Beschl. v. 24.3.2021 - 1 BvR 2656/18, 1 BvR 78/20, 1 BvR 96/20, 1 BvR 288/20, Rdnr. 246.

43) BVerfG, Beschl. v. 24.3.2021 - 1 BvR 2656/18, 1 BvR 78/20, 1 BvR 96/20, 1 BvR 288/20, Rdnr. 218. 
letzung ihrer Grundrechte mit Blick auf die Artt. 2 Abs. 2 S. 1 und 14 GG nicht aus. Denn aus diesen Grundrechten resultieren über deren Charakter als bloße Abwehr staatlicher Eingriffe hinausgehend jedenfalls staatliche Schutzpflichten, die sich auch gegen Beeinträchtigungen durch Umweltbelastungen bzw. den Klimawandel zu richten haben. Bei den Maßgaben des KSG zur Reduzierung der $\mathrm{CO}_{2}$-Emissionen könnte es sich immerhin um einen Beitrag zur Ausformung dieser Schutzpflicht handeln. ${ }^{44}$ Außerdem sieht das Gericht wegen auf die Bf. nach 2030 zukommenden ,sehr hohen (Treibhausgas-)Minderungslasten“ die Möglichkeit einer Verletzung ihrer ,grundrechtlich umfassend geschützten Freiheit, weil das KSG erhebliche Anteile der durch Art. 20a GG gebotenen Treibhausgasminderungslasten auf Zeiträume nach 2030 verschiebt" ${ }^{\text {" } 45}$. Da aber nahezu alle Bereiche menschlichen Lebens mit der Emission von Treibhausgasen verbunden seien, könnte es dann notwendig werden, weitere Reduzierungslasten so kurzfristig zu erbringen, dass den Bf. enorme Anstrengungen abverlangt würden, die ihre grundrechtlich geschützte Freiheit umfassend bedrohten. ${ }^{46}$ So gesehen seien die notwendigen Freiheitsbeschränkungen der Zukunft bereits in den Großzügigkeiten des gegenwärtigen Klimaschutzes angelegt. ${ }^{47}$ Darauf bezogen hält das Gericht potenziell eine Betroffenheit jeglicher Freiheit, im Mindesten aber die der in Art. 2 Abs. 1 GG verorteten allgemeinen Handlungsfreiheit als dem ,grundlegenden Freiheitsrecht", für nicht ausgeschlossen. ${ }^{48}$ Es erscheine jedenfalls möglich, dass die Grundrechte des GG als ,,intertemporale Freiheitssicherung" vor Regelungen schützen, die mit Blick auf das Deutschland noch zustehende Restbudget einen vorzeitigen und nach derzeitigem Stand irreversiblen Verbrauch der danach verbleibenden $\mathrm{CO}_{2}$-Mengen zulassen, ohne dabei hinreichend Rücksicht auf die hierdurch gefährdete künftige Freiheit zu nehmen. ${ }^{49}$ Bei dieser Freiheit gehe es aber - so das Gericht klarstellend - ausschließlich um diejenige der Bf., ${ }^{50}$ nicht etwa auch um die Rechte noch ungeborener Menschen. Denn künftige, noch nicht lebende Generationen können keine Grundrechtsträger sein; würde doch eine derartige Vorverlagerung des Grundrechtsschutzes mangels eines tauglichen Grundrechtssubjekts ins Leere gehen. Die Berücksichtigung der Belange der künftigen Generationen mit Blick auf den Umweltschutz im Allgemeinen und den Klimaschutz im Besonderen finden Berücksichtigung im Rahmen des Art. 20a GG. Überdies wird die gegenwärtige Grundrechtsbetroffenheit der Bf. bejaht, weil die Gefahr künftiger Freiheitsbeschränkung - gleichsam vorwirkend - ,im aktuellen Recht angelegt" sei. ${ }^{51}$ Dass die $\$ 3$ Abs. 1 S. 2 und $\$ 4$ Abs. S. 3 KSG i. V.m. Anlage 2 Treibhausgasemissionen bis 2030 in einem möglicherweise zu hohen Umfang zulassen, bedrohe nämlich alle nach 2030 mit $\mathrm{CO}_{2}$-Emissionen verbundenen Freiheitsausübungen. Denn wegen der nach aktuellem wissenschaftlichem Erkenntnisstand fehlenden Möglichkeit, einmal in die Erdatmosphäre gelangte $\mathrm{CO}_{2}$-Emissionen wieder in großem Umfang entnehmen zu können, würden bereits heute möglicherweise unumkehrbar Grundrechtsbeeinträchtigungen in Gang gesetzt, die mit einer späteren Verfassungsbeschwerde nicht mehr ohne Weiteres erfolgreich angegriffen werden könnten. ${ }^{52}$

\subsection{Begründetheitsfragen}

In der Begründetheitsprüfung befasst sich das Gericht sodann mit den Fragen einer möglichen Verletzung von Schutzpflichten und den an eine ,intertemporale Freiheitssicherung " zu stellenden Anforderungen.

\subsubsection{Schutzpflichten}

So werden aufgrund der Gefahren des Klimawandels allem voran Schutzverpflichtungen des Staates gegenüber den (in Deutschland) lebenden Bf. aus Art. 2 Abs. 2 S. 1 und Art. 14 Abs. 1 GG bejaht. ${ }^{53}$ Bei diesen Schutzpflichten handele es sich grundsätzlich um einen Teil der ,subjektiven Grundrechtsberechtigung“; weswegen in einer Schutzpflichtverletzung zugleich auch stets eine Verletzung des Grundrechts liege. ${ }^{54}$

\subsubsection{Schutzpflichten aus Art. 2 Abs. 2 S. 1 GG}

Mit Blick auf den zunächst in Bezug genommenen Art. 2 Abs. 2 S. 1 GG stellt das Gericht - in Fortsetzung seiner st. Rspr. - klar, dass dieser nicht nur als subjektives Abwehrrecht gegen staatliche Eingriffe schütze, sondern auch die Pflicht des Staates impliziere, sich schützend und fördernd vor die Rechtsgüter Leben und körperliche Unversehrtheit $\mathrm{zu}$ stellen und sie vor rechtswidrigen Eingriffen von Seiten anderer zu bewahren; was auch deren ,Schutz vor Beeinträchtigungen und insbesondere vor Schädigungen grundrechtlicher Schutzgüter durch Umweltbelastungen“55 einschließe, ,gleich von wem und durch welche Umstände sie drohen" ${ }^{66}$. Entsprechendes habe hinsichtlich der vom Klimawandel ausgehenden Gefahren zu gelten. ${ }^{57}$ Dem stehe nicht entgegen, dass ,der deutsche Staat diesen Klimawandel wegen der globalen Wirkung und des globalen Charakters seiner Ursache nicht allein, sondern nur in internationaler Einbindung anhalten kann“; im Gegenteil erfordere der Schutzpflichtenrahmen des Art. 2 Abs. 2 S. 1 GG insoweit ,ein international ausgerichtetes Handeln zum globalen Schutz des Klimas“ etwa durch entsprechende völkerrechtliche Vereinbarungen. ${ }^{58}$ Dies dürfte in dieser Grundsätzlichkeit zwar kaum zu beanstanden sein, gleichwohl stellt sich die Frage, wie bzw. inwieweit die grundrechtliche Schutzpflicht sich vor diesem Hintergrund realistischerweise überhaupt konkretisieren ließe. Sie würde dann eben nicht (nur) von den tatsächlichen Erfordernissen des globalen wie nationalen Klimaschutzes, sondern realistischerweise an dem jeweils politisch Erreichbaren zu messen sein; für die Erfüllung einer Rechtspflicht ein wohl ziemlich vager Maßstab. Überdies stellt sich die Frage, ob die vom BVerfG hier bejahte Reduzierungsverpflichtung auch dann bestehen kann bzw. darf, wenn die überwiegende Zahl der anderen Vertragsstaaten des Pariser Klimaschutzabkommens nicht oder viel zu wenig unternimmt, um das Problem

44) BVerfG, Beschl. v. 24.3.2021 - 1 BvR 2656/18, 1 BvR 78/20, 1 BvR 96/20, 1 BvR 288/20, Rdnr. 96 ff.

45) BVerfG, Beschl. v. 24.3.2021 - 1 BvR 2656/18, 1 BvR 78/20, 1 BvR 96/20, 1 BvR 288/20, Rdnr. 96.

46) BVerfG, Beschl. v. 24.3.2021 - 1 BvR 2656/18, 1 BvR 78/20, 1 BvR 96/20, 1 BvR 288/20, Rdnr. 117.

47) BVerfG, Beschl. v. 24.3.2021 - 1 BvR 2656/18, 1 BvR 78/20, 1 BvR 96/20, 1 BvR 288/20, Rdnr. 120.

48) BVerfG, Beschl. v. 24.3.2021 - 1 BvR 2656/18, 1 BvR 78/20, 1 BvR 96/20, 1 BvR 288/20, Rdnr. 117.

49) BVerfG, Beschl. v. 24.3.2021 - 1 BvR 2656/18, 1 BvR 78/20, 1 BvR 96/20, 1 BvR 288/20, Rdnr. 122.

50) BVerfG, Beschl. v. 24.3.2021 - 1 BvR 2656/18, 1 BvR 78/20, 1 BvR 96/20, 1 BvR 288/20, Rdnr. 131.

51) BVerfG, Beschl. v. 24.3.2021 - 1 BvR 2656/18, 1 BvR 78/20, 1 BvR 96/20, 1 BvR 288/20, Rdnr. 130.

52) BVerfG, Beschl. v. 24.3.2021 - 1 BvR 2656/18, 1 BvR 78/20, 1 BvR 96/20, 1 BvR 288/20, Rdnr. 130.

53) BVerfG, Beschl. v. 24.3.2021 - 1 BvR 2656/18, 1 BvR 78/20, 1 BvR 96/20, 1 BvR 288/20, Rdnr. 143 ff.

54) BVerfG, Beschl. v. 24.3.2021 - 1 BvR 2656/18, 1 BvR 78/20, 1 BvR 96/20, 1 BvR 288/20, Rdnr. 145.

55) BVerfG, Beschl. v. 24.3.2021 - 1 BvR 2656/18, 1 BvR 78/20, 1 BvR 96/20, 1 BvR 288/20, Rdnr. 145.

56) BVerfG, Beschl. v. 24.3.2021 - 1 BvR 2656/18, 1 BvR 78/20, 1 BvR 96/20, 1 BvR 288/20, Rdnr. 147.

57) BVerfG, Beschl. v. 24.3.2021 - 1 BvR 2656/18, 1 BvR 78/20, 1 BvR 96/20, 1 BvR 288/20, Rdnr. 148.

58) BVerfG, Beschl. v. 24.3.2021 - 1 BvR 2656/18, 1 BvR 78/20, 1 BvR 96/20, 1 BvR 288/20, Rdnr. 149. 
des $\mathrm{CO}_{2}$-Ausstoßes zu bewältigen, sodass die eigenen Anstrengungen nutzlos bleiben. ${ }^{59}$ Angesichts des Umstandes, dass zuletzt lediglich 32 der 191 Vertragsstaaten des Pariser Klimaschutzabkommens tatsächlich Reduzierungszusagen getroffen haben und andere ( $z$. B die zu den größten globalen Emittenten gehörenden Staaten China und Indien) ihre Emissionen sogar noch steigern wollen, ist dies auch beileibe kein bloß theoretisches Gedankenspiel.

Zudem verpflichte Art. 2 Abs. 2 S. 1 GG den Staat, soweit der Klimawandel nicht aufgehalten werden kann oder bereits eingetreten ist, den Gefahren durch positive Schutzmaßnahmen (Anpassungsmaßnahmen) zu begegnen und in seinen negativen Folgen auf ein ,,verfassungsrechtlich hinnehmbares Maß zu begrenzen" 60 . Hier bleibt bereits unklar, wie überhaupt erkannt werden soll, ob etwas als Folge des (durch Menschen verursachten) Klimawandels oder aufgrund immer wieder auftretender Witterungsanomalien eingetreten ist? Was zudem eine Begrenzung von $\mathrm{Ne}-$ gativfolgen auf ein verfassungsrechtlich hinnehmbares Maß bedeuten soll, erschließt ebenfalls nicht.

Des Weiteren greife diese staatliche Schutzpflicht nach den Vorstellungen des Gerichts nicht erst bzw. nur dann ein, wenn Verletzungen bereits eingetreten sind, sie sei vielmehr auch auf die Zukunft gerichtet und könne insoweit auch eine Schutzverpflichtung in Bezug auf künftige Generationen begründen; was erst recht gelte, wenn - wie im Falle drohender negativer Folgen des Klimawandels - unumkehrbare Entwicklungen in Rede stehen. Dieser ,,intergenerationellen Schutzverpflichtung " komme allerdings allein ,objektivrechtliche“"Natur zu, weil künftig lebende Menschen nicht ,grundrechtsfähig“" seien. ${ }^{61}$ Das irritiert zumindest: Denn wenn Schutzmechanismen gleich ob subjektiv- oder objektivrechtlicher Natur - dem Art. 2 Abs. 2 S. 1 GG immanent sein sollen, müssen deren Schutzadressaten zumindest grundrechtsfähig, also derzeit lebende Menschen sein. Hier scheint das Gericht die für künftige Generationen insoweit eigentlich nur aus Art. 20a GG herleitbaren Schutzpflichten ohne Not systemwidrig in den Art. 2 Abs. 2 S. 1 GG transformieren zu wollen. Der zusätzliche Nutzen, den eine solche rein objektive, also für niemanden gegenüber dem Staat einforderbare Obliegenheit angesichts eines ohnehin bestehenden gleichartigen Schutzpostulats in Art. 20a GG haben könnte, bleibt dabei jedenfalls im Dunkeln.

Allerdings - so das Gericht - sei eine derartige Schutzpflicht wegen ihrer grundsätzlichen Unbestimmtheit nur begrenzt verfassungsgerichtlich überprüfbar; insoweit sei , die Entscheidung, in welcher Weise Gefahren entgegengewirkt werden soll, die Aufstellung eines Schutzkonzepts und dessen normative Umsetzung ... Sache des Gesetzgebers, dem auch dann ein Einschätzungs-, Wertungs- und Gestaltungsspielraum zukommt", wenn er dem Grunde nach zum Ergreifen von Maßnahmen verpflichtet ist. ${ }^{62}$

Nur - und damit bleibt das BVerfG seiner bisherigen Rechtsprechungslinie treu ${ }^{63}$ - wenn Schutzvorkehrungen entweder überhaupt nicht getroffen, die getroffenen Reglungen und Maßnahmen offensichtlich ungeeignet oder unzulänglich sind, um das gebotene Schutzziel zu erreichen, oder wenn sie erheblich hinter dem Schutzziel zurück bleiben, könne diese Schutzpflicht verletzt sein. ${ }^{64}$ Eine solche Verletzung sieht das BVerfG hier aber ,im Ergebnis“ ausdrücklich nicht. ${ }^{65}$ Es attestiert dem Gesetzgeber unter ausführlicher Befassung mit den von den Bf. als unzureichend beanstandeten gesetzgeberischen Klimaschutzbemühungen im KSG, Schutzvorkehrungen getroffen zu haben, die nicht offensichtlich ungeeignet sind, zumal dieser ja gerade mit den angegriffenen Bestimmungen des KSG Anstrengungen zur Begrenzung des Klimawandels unternommen habe. ${ }^{66}$ Überhaupt anerkennt das Gericht, dass der Gesetzgeber mit dem KSG und anderen Gesetzen den Ausstoß von Treibhausgasemissionen beschränkt. ${ }^{67}$ Letztlich lasse sich nicht feststellen, dass die angegriffenen Reg- lungen erheblich hinter dem durch Art. 2 Abs. 2 S. 1 GG gebotenen Schutz zurück blieben. ${ }^{6}{ }^{6}$ Konkrete Maßnahmen sind daher auch im Klimaschutz nicht einklagbar. ${ }^{69}$

\subsubsection{Schutzpflichten aus Art. 14 Abs. 1 GG}

Des Weiteren prüft das BVerfG hinsichtlich des infolge des Klimawandels möglicherweise Schaden nehmenden Eigentums zurecht, ob die aus Art. 14 Abs. 1 GG resultierende staatliche Schutzpflicht durch den Gesetzgeber des KSG verletzt ist. Dies wird aber ebenso wie schon mit Blick auf Art. 2 Abs. 2 S. 1 GG verneint. ${ }^{70}$ Es sei derzeit nicht absehbar, dass in Deutschland belegenes Eigentum so sehr gefährdet würde, dass dies nicht durch Schutzmaßnahmen in verfassungsrechtlichen vertretbaren Maßen gehalten werden könnte.

\subsubsection{Schutzpflichten gegenüber im Ausland Lebenden}

Das BVerfG bejaht zwar auch eine prinzipiell bestehende grundrechtliche Schutzpflicht gegenüber im Ausland (hier in Bangladesch und Nepal) lebenden Bf., ${ }^{71}$ weist aber zugleich zurecht darauf hin, dass diese Schutzpflicht aufgrund beschränkter Aktionsoptionen Deutschlands im Ausland lediglich geringeren grundrechtlichen Anforderungen unterliegen werde als jene, die gegenüber innerhalb Deutschlands lebenden Menschen zu erfüllen sei. ${ }^{72}$ Auch im Ergebnis gut vertretbar wird nach eingehender Erörterung eine Schutzpflichtenverletzung verneint, weil die deutsche Staatsgewalt , durch das internationale Eintreten für den Klimaschutz und durch konkrete Maßnahmen zur Umsetzung des international zum Klimaschutz Vereinbarten genügt ${ }^{\text {"73 }}$.

\subsection{2 „Intertemporale Freiheitssicherung“}

Das BVerfG hält die ,in $₫ 3$ Abs. 1 S. 2 und $\$ 4$ Abs. 1 S. 3 KSG i. V.m. Anlage 2 getroffene Reglung der bis zum Jahr 2030 zulässigen Emissionsmengen mit Blick auf die hierdurch in späteren Reduktionsphasen erheblich gefährdete Freiheit ... ohne weitere Vorkehrungen" für verfassungswidrig. ${ }^{74}$ Es sieht in den genannten Paragraphen zwar keinen Verstoß gegen das Klimaschutzgebot des Art. 20a

59) So Murswiek, in: Welt v. 29.8.2021, Stand 12.12.2021, abrufbar unter https://www.welt.de/wirtschaft/plus233215175/KlimaUrteil-Oekonomisch-und-oekologisch-unsinnig-verfassungsrechtlich-falsch.html.

60) BVerfG, Beschl. v. 24.3.2021 - 1 BvR 2656/18, 1 BvR 78/20, 1 BvR 96/20, 1 BvR 288/20, Rdnr. 150, 164.

61) BVerfG, Beschl. v. 24.3.2021 - 1 BvR 2656/18, 1 BvR 78/20, 1 BvR 96/20, 1 BvR 288/20, Rdnr. 146, vgl. auch Rdnr. 148.

62) BVerfG, Beschl. v. 24.3.2021 - 1 BvR 2656/18, 1 BvR 78/20, 1 BvR 96/20, 1 BvR 288/20, Rdnr. 152.

63) BVerfG, Beschl. v. 26.7.2016 - 1 BvL 8/15, BVerfGE 142, 313, 337 f. m. w. N.

64) BVerfG, Beschl. v. 24.3.2021 - 1 BvR 2656/18, 1 BvR 78/20, 1 BvR 96/20, 1 BvR 288/20, Rdnr. 152.

65) BVerfG, Beschl. v. 24.3.2021 - 1 BvR 2656/18, 1 BvR 78/20, 1 BvR 96/20, 1 BvR 288/20, Rdnr. 153.

66) BVerfG, Beschl. v. 24.3.2021 - 1 BvR 2656/18, 1 BvR 78/20, 1 BvR 96/20, 1 BvR 288/20, Rdnr. 154-156.

67) BVerfG, Beschl. v. 24.3.2021 - 1 BvR 2656/18, 1 BvR 78/20, 1 BvR 96/20, 1 BvR 288/20, Rdnr. 157.

68) BVerfG, Beschl. v. 24.3.2021 - 1 BvR 2656/18, 1 BvR 78/20, 1 BvR 96/20, 1 BvR 288/20, Rdnr. 158

69) Frenz, DÖV 2021, 715, 717.

70) BVerfG, Beschl. v. 24.3.2021 - 1 BvR 2656/18, 1 BvR 78/20, 1 BvR 96/20, 1 BvR 288/20, Rdnr. $171 \mathrm{f}$.

71) BVerfG, Beschl. v. 24.3.2021 - 1 BvR 2656/18, 1 BvR 78/20, 1 BvR 96/20, 1 BvR 288/20, Rdnr. $173 \mathrm{ff}$.

72) BVerfG, Beschl. v. 24.3.2021 - 1 BvR 2656/18, 1 BvR 78/20, 1 BvR 96/20, 1 BvR 288/20, Rdnr. 178.

73) BVerfG, Beschl. v. 24.3.2021 - 1 BvR 2656/18, 1 BvR 78/20, 1 BvR 96/20, 1 BvR 288/20, Rdnr. 181.

74) BVerfG, Beschl. v. 24.3.2021 - 1 BvR 2656/18, 1 BvR 78/20, 1 BvR 96/20, 1 BvR 288/20, Rdnr. 175, 243. 
GG, hat insoweit also „,keine durchgreifenden Bedenken“, doch sei die mit den betreffenden $\mathrm{CO}_{2}$-Emissionsmengenregelungen einhergehende Grundrechtsvorwirkung „,verfassungsrechtlich nicht vollständig zu rechtfertigen" ${ }^{775}$ " unten 4.2.2.2). Denn die in Rede stehenden KSG-Regelungen vermögen die derzeit durch sie nur unzureichend eingedämmte Gefahr schwerwiegender Grundrechtsbeeinträchtigungen in der Zukunft nicht abzuwenden. ${ }^{76}$ Mit anderen Worten: die aktuell ergriffenen Maßnahmen zur $\mathrm{CO}_{2}-\mathrm{Re}-$ duzierung reichten nicht aus, um in späterer Zeit die jetzt jungen Menschen vor weitaus größeren Reduzierungsanstrengen zu bewahren, was deren Freiheitsgebrauch dann unangemessen stark beschränken würde. Dadurch sieht das Gericht das bereits an anderer Stelle (oben 4.1) erwähnte, aus den Freiheitsgrundrechten, insbesondere aber aus dem Grundrecht auf allgemeine Handlungsfreiheit (Art. 2 Abs. 1 GG) resultierende Gebot der ,,intertemporalen Freiheitssicherung" der (in Deutschland lebenden) Bf. verletzt. Der Gesetzgeber müsse nämlich ,,zur Gewährleistung eines freiheitsschonenden Übergangs in die Klimaneutralität hinreichende Vorkehrungen treffen“. Dabei verpflichte das GG ,unter bestimmten Voraussetzungen zur Sicherung grundrechtsgeschützter Freiheit über die Zeit und zur verhältnismäßigen Verteilung von Freiheitschancen über die Generationen" "77.

\subsubsection{1 „Eingriffsähnliche Vorwirkung“}

Bemerkenswert an der Argumentation des BVerfG ist die von ihm in diesem Rahmen vorgenommene Konstruktion einer in der Grundrechtsdogmatik bisher unbekannten „eingriffsähnlichen Vorwirkung“, welche die von den Bf. beanstandeten KSG-Vorschriften auf die durch das GG umfassend geschützte Freiheit entfalteten. ${ }^{78}$ Diese bedürfe einer verfassungsrechtlichen Rechtfertigung (dazu unten 4.2.2.2). Dem stehe zwar die von der Verfassung ebenfalls durch die Freiheitsgrundrechte geschützte ,aktuell noch hohe Zahl an Verhaltensweisen des täglichen Lebens, des Arbeitens und des Wirtschaftens“ gegenüber, „die ... dazu führen, dass $\mathrm{CO}_{2}$-Emissionen in die Erdatmosphäre gelangen" ${ }^{79}$. Doch unterliegen diese - so das Gericht weiter - ,den vom Gesetzgeber zum Schutz des Klimas nach Art. 20a GG wie auch zur Erfüllung grundrechtlicher Schutzpflichten zu ziehenden Grenzen" ${ }^{\circ 80}$.

Im Hinblick darauf unterstellt das Gericht pauschal, dass alle heute $\mathrm{CO}_{2}$-Emissionen zulassenden Vorschriften ,eine unumkehrbar angelegte rechtliche Gefährdung künftiger Freiheit" begründeten, weil mit jeder $\mathrm{CO}_{2}$-Emissionsmenge „das verfassungsrechtlich vorgezeichnete Restbudget (oben 3) irreversibel verkleinert und $\mathrm{CO}_{2}$-relevanter Freiheitsgebrauch stärkeren, verfassungsrechtlich gebotenen Restriktionen ausgesetzt sein" werde. Insbesondere ein schneller Verbrauch des $\mathrm{CO}_{2}$-Restbudgets schon bis 2030 verschärfe das Risiko schwerwiegender Freiheitseinbußen, weil damit die Zeitspanne für technische und soziale Entwicklungen knapper werde, mit deren Hilfe die Umstellung von der heute noch umfassend mit $\mathrm{CO}_{2}$-Emissionen verbundenen Lebensweise auf klimaneutrale Verhaltensweisen freiheitsschonend vollzogen werden könnte. ${ }^{81}$ Dabei betont das Gericht, dass es das Verfassungsrecht selbst (gemeint ist wiederum Art. 20a GG) sei, das mit jedem Anteil, der vom endlichen $\mathrm{CO}_{2}$-Restbudget verzehrt werde, umso dringlicher aufgebe, weitere $\mathrm{CO}_{2}$-relevante Freiheitsausübung zu unterbinden. Dies erscheint schon deshalb fragwürdig, weil es sich bei dem hiesigen $\mathrm{CO}_{2}$-Restbudget aufgrund seiner fehlenden Verankerung im Verfassungsrecht (oben 3) gerade nicht um einen verfassungsrechtlich bindenden Parameter, damit auch nicht um eine Grundrechtseingriffe rechtfertigende Vorgabe mit Verfassungsrang handeln kann. Diesen simplen Sachverhalt übergeht das BVerfG vollständig. Gerade dieses $\mathrm{CO}_{2}$-Restbudget ist es jedoch, welches das Gericht erst dazu verleitet, eine Gefahr künftiger Freiheitseinbußen annehmen zu sollen. Dieses Budget vermag aber allenfalls einen (verfassungs)rechtlich unverbindlichen Orientierungsmaßstab darzustellen (oben 3). Keinesfalls darf es indes - wie vom Gericht praktiziert ${ }^{82}$ - zu den staatliches Eingriffshandeln rechtfertigenden ,elementaren Grundentscheidungen und allgemeinen Verfassungsgrundsätzen des GG“ gezählt werden, an denen die Verfassungsmäßigkeit der in Rede stehenden KSG-Vorschriften zu messen sei.

Hinzu kommt, dass das Gericht dem Gesetzgeber an anderer Stelle gerade ausdrücklich zubilligt, , $\left(\mathrm{CO}_{2}^{-}\right)$Minderungsziele zu formulieren, ohne dabei von Beginn an eine Vorstellung davon zu entwickeln, welche Gesamtemissionsmenge noch zur Verfügung steht" und ermöglicht ihm damit eine Entkoppelung des Restbudgets von den zu treffenden $\mathrm{CO}_{2}$-Reduzierungsmaßnahmen (vgl. auch oben 3). ${ }^{83}$ Dies bedeutete letztlich, dass der Gesetzgeber sich als Maßstab für sein Vorgehen gerade nicht an dem ohnehin (selbst mit noch so rigiden staatlichen Maßnahmen) realistischerweise wohl nicht (mehr) einhaltbaren und damit eine bloße Utopie verkörpernden $\mathrm{CO}_{2}$-Emissionsrestbudget für Deutschland orientieren muss, sondern allein an dem zur Erreichung des das Klimaschutzgebot des Art. 20a GG konkretisierenden „Paris-Zieles" maßgeblichen Zeitraum bis 2050; immerhin eine insoweit verbleibende Zeitspanne von zwei Jahrzehnten. Im Übrigen verstünde es sich von selbst, dass vom Staat nichts verlangt werden kann, was jenseits seiner (verfassungs)rechtlichen wie tatsächlichen Möglichkeiten liegt.

Dass überdies „, $\mathrm{CO}_{2}$-Emissionen nach dem derzeitigen Stand im Wesentlichen unumkehrbar zur Erwärmung der Erde beitragen" ${ }^{84}$, erscheint aber keineswegs als tatsächlich gesichert. Schon die Inbezugnahme des derzeitigen Erkenntnisstandes reduziert zumindest die durch die $\mathrm{CO}_{2}$ Emissionen bewirkte Unumkehrbarkeit letztlich in ihrer Bedeutung auf die einer aktuellen Momentaufnahme. Dass die $\mathrm{CO}_{2}$-Emissionen einen Beitrag zur Erderwärmung zu leisten vermögen, soll gar nicht bestritten werden. $\mathrm{Ob}$ aber einmal in der Atmosphäre eingelagertes $\mathrm{CO}_{2}$ tatsächlich irreversibel in der Atmosphäre eingelagert bleibt bzw. bleiben muss, dürfte keineswegs ausgemacht sein. Ungeachtet dessen bedeutet „Beitrag zur Erderwärmung“ doch wohl, dass es offenbar eine Anzahl anderer wichtiger, vom Gericht aber überhaupt nicht gewürdigte Faktoren gibt, die ebenso für die Erderwärmung mitursächlich sind, wie etwa der Ausstoß anderer Treibhausgase, die großflächige Abholzung von Wäldern oder die übermäßige Nutzung natürlicher Ressourcen. Wie groß indes der alleinige Beitrag der anthropogenen $\mathrm{CO}_{2}$-Emissonen an der Erderwärmung tatsächlich ist, lässt sich überdies redlicherweise kaum ermessen. Allein schon die Vorgehensweise, eine von mehreren Ursachen für die Erderwärmung exklusiv herauszuheben und dies als Rechtfertigung für Einschränkungen des grundrechtlichen Freiheitsgebrauchs zu legitimieren, erscheint bedenklich.

75) BVerfG, Beschl. v. 24.3.2021 - 1 BvR 2656/18, 1 BvR 78/20, 1 BvR 96/20, 1 BvR 288/20, Rdnr. 195.

76) BVerfG, Beschl. v. 24.3.2021 - 1 BvR 2656/18, 1 BvR 78/20, 1 BvR 96/20, 1 BvR 288/20, Rdnr. 195, 243.

77) BVerfG, Beschl. v. 24.3.2021 - 1 BvR 2656/18, 1 BvR 78/20, 1 BvR 96/20, 1 BvR 288/20, Rdnr. 183.

78) BVerfG, Beschl. v. 24.3.2021 - 1 BvR 2656/18, 1 BvR 78/20, 1 BvR 96/20, 1 BvR 288/20, Rdnr. 183 u. 184.

79) BVerfG, Beschl. v. 24.3.2021 - 1 BvR 2656/18, 1 BvR 78/20, 1 BvR 96/20, 1 BvR 288/20, Rdnr. 184.

80) BVerfG, Beschl. v. 24.3.2021 - 1 BvR 2656/18, 1 BvR 78/20, 1 BvR 96/20, 1 BvR 288/20, Rdnr. 185

81) BVerfG, Beschl. v. 24.3.2021 - 1 BvR 2656/18, 1 BvR 78/20, 1 BvR 96/20, 1 BvR 288/20, Rdnr. 186, 187.

82) Vgl. BVerfG, Beschl. v. 24.3.2021 - 1 BvR 2656/18, 1 BvR 78/20, 1 BvR 96/20, 1 BvR 288/20, Rdnr. $188 \mathrm{ff}$.

83) BVerfG, Beschl. v. 24.3.2021 - 1 BvR 2656/18, 1 BvR 78/20, 1 BvR 96/20, 1 BvR 288/20, Rdnr. 218.

84) BVerfG, Beschl. v. 24.3.2021 - 1 BvR 2656/18, 1 BvR 78/20, 1 BvR 96/20, 1 BvR 288/20, Rdnr. 185. 
Nicht zuletzt auch deshalb ist es bemerkenswert, dass das Gericht die Freiheitsrechte der jetzt in Deutschland lebenden Menschen (dazu gehören übrigens „Alt“ und „Jung“" gleichermaßen!) gegen eine wie auch immer auszugestaltende ,künftige“"Freiheit setzt. Zeichnen doch derzeit generationenübergreifend alle Menschen mehr oder weniger für $\mathrm{CO}_{2}$-Emissionen verantwortlich, nehmen also im Sprachgebrauch des BVerfG ,„CO $\mathrm{CO}_{2}$-relevanten Freiheitsgebrauch" für sich in Anspruch. Zum einen stellt sich die Frage, wie hoch dann überhaupt der aktuelle $\mathrm{CO}_{2}$-relevante Freiheitsgebrauch des Einzelnen noch ausfallen darf? Zum anderen erscheint es nicht unproblematisch, auf der Grundlage wie auch immer gearteter Prognosen und Erwartungen die künftige gegen die aktuelle Freiheit auszuspielen. Mit der vom BVerfG gebrauchten Argumentation ließe sich staatlicherseits jedenfalls ziemlich beliebig freiheitsbeschränkend in die Grundrechte eingreifen. Was vor diesem Hintergrund die grundrechtlich verbürgte Freiheitsausübung tatsächlich noch für einen Wert hätte, lässt sich nur schwer erahnen. Abgesehen von der Beliebigkeit der dann möglichen staatlichen „Eingriffe“ stellte sich in concreto die Frage, welche Anforderungen an eine verfassungsrechtlich zu rechtfertigende Einschränkung aktueller grundrechtlicher Freiheit dann jeweils zu gelten hätten.

Überhaupt zeigt sich das BVerfG beim Klimaschutz ersichtlich von der Maxime beseelt, dass höhere Belastungen der Jetzigen unabdingbar seien, damit deren Belastungen später umso geringer ausfallen können. ${ }^{85}$ So leitet es aus dem Grundsatz der Verhältnismäßigkeit eine Art „Generationengerechtigkeitsprinzip“ her, in dem es meint, ,dass nicht einer Generation zugestanden werden darf, unter vergleichsweise milder Reduktionslast große Teile des $\mathrm{CO}_{2}$-Budgets zu verbrauchen, wenn damit zugleich nachfolgenden Generationen eine - von den Bf. als ,Vollbremsung' bezeichnete - radikale Reduktionslast überlassen und deren Leben schwerwiegenden Freiheitseinbußen ausgesetzt würde“. Dementsprechend seien die nach Art. 20a GG verfassungsrechtlich notwendigen $\mathrm{CO}_{2}$-Emissionsreduzierungen ,,bis hin zur Klimaneutralität vorausschauend so zu gestalten, dass die damit verbundenen Freiheitseinbußen trotz steigender Klimaschutzanforderungen weiterhin zumutbar ausfallen und die Reduktionslasten über die Zeit und zwischen den Generationen nicht einseitig zulasten der Zukunft verteilt sind" $" 86$.

Hierin mag - zumindest vordergründig betrachtet durchaus eine gewisse Logik begründet liegen. Doch dürfte in dieser von richterlichem ,worst-case-Szenarien“ geprägten Sichtweise bei genauerem Hinschauen eine juristisch kaum fassbare Pauschalisierung zum Ausdruck kommen. Stellt sich doch allem voran die Frage, nach der tatsächlichen Messbarkeit der jeweils ,generationengerecht“ zu verteilenden Lasten. Anders ausgedrückt: Wie könnte gegebenenfalls eine generationengerechte Lastenverteilung aussehen? Konkreter: Wie ließe sich denn feststellen, welche Generation tatsächlich die Hauptlast mit Blick auf notwendige $\mathrm{CO}_{2}$-Reduzierungen $\mathrm{zu}$ tragen hätte? Welche Maßstäbe gäbe es gegebenenfalls dafür, um eine unangemessene Lastenverteilung zu vermeiden? Wäre es etwa angemessen bzw. "gerecht" der jetzigen Generation mehr aufzubürden als den künftigen? Oder gibt es gar das verfassungsrechtliche Gebot, jetzige wie künftige Generationen in der Verminderung der Reduzierungslasten gleich zu behandeln? - Fragen über Fragen, die auf Antworten warten.

Dabei erscheint schon die geradezu stereotyp bemühte Prämisse, dass zurzeit die Anstrengungen zur $\mathrm{CO}_{2}-\mathrm{Redu}-$ zierung in Deutschland zu moderat ausfielen, für sich genommen eine mit nur wenigen Fakten unterlegte Annahme zu sein. Dies hat umso mehr zu gelten, als bislang der Ablauf des Wandels und seiner damit einhergehenden Folgen nur schwer präzise vorhersehbar sind. Obendrein ist es keineswegs ausgemacht, dass die vom BVerfG angenommenen $\mathrm{CO}_{2}$-reduzierungsbedingten Lasten mit Blick auf die grundrechtsrelevanten Beeinträchtigungen künftig tat- sächlich höher ausfallen werden (müssen) als die aktuellen. Bleibt doch in den Erwägungen des Gerichts der im Zuge des technologischen und naturwissenschaftlichen Fortschritts zu erwartende gesamtgesellschaftliche Verhaltenswandel und die damit einhergehenden Möglichkeiten, den nachteiligen Folgen des Wandels angemessen begegnen zu können, unberücksichtigt. Ebenso unterlässt es das Gericht, auch wirtschaftliche Modelle und Innovationen für die $\mathrm{Zu}$ kunftsgestaltung ins Kalkül zu ziehen. Ein Blick in die Vergangenheit etwa zeigt, dass die $\mathrm{CO}_{2}$-Emissionen seit 1990 bereits erhebliche Reduzierungen ( $55 \%$ ) erfahren haben und die Reduzierungen auch jetzt weiter vorangetrieben werden. Dies geschah bisher unter den gegebenen und jeweils realistisch möglichen Voraussetzungen, wie eben fortan mit dem sich anbahnenden „Ausstieg“ aus den fossilen Energieträgern erhebliche $\mathrm{CO}_{2}$-Reduzierungsmaßnahmen ergriffen werden. Es darf überdies immerhin davon ausgegangen werden, dass durch die schon aktuell weit vorangeschrittene Sensibilisierung der Politik und weiter Bevölkerungskreise auch fortan Anstrengungen unternommen werden. Insbesondere wird vom BVerfG die längst nicht auszuschließende Möglichkeit übergangen, dass der besagte Fortschritt dafür sorgte, dass die jetzt Lebenden im Laufe der Zeit überhaupt keinen besonderen $\mathrm{CO}_{2}$-Reduzierungslasten mehr ausgesetzt sein könnten, außer denen, die durch dann etablierte Technik ohnehin bestehen. Zudem bleibt unbeachtet, dass nicht für jede pro Jahr reduzierte Tonne $\mathrm{CO}_{2}$ künftig ein immer gleich intensiver Einschränkungsaufwand erforderlich sein wird. Vor diesem Hintergrund ließe sich aber nicht einmal sicher ausschließen, dass tatsächlich schon derzeit die $\mathrm{CO}_{2}$-reduzierungsrelevante Hauptlast getragen wird. Alles in allem handelt es sich bei dem vom Gericht betonten verhältnismäßigen Schutz der jüngeren Menschen mit Blick auf deren vermeintlich bedrohten Zukunftschancen gewiss um ein löbliches Anliegen, das allerdings den ebenfalls grundrechtlich unterlegten Anspruch aller hier und jetzt lebenden Menschen, vor unverhältnismäßigen staatlichen Eingriffen geschützt zu werden, nicht so ohne weiteres gerecht zu werden vermag. Dies gilt umso mehr, wenn eine bislang allenfalls vermeintlich feststehende künftige Belastung in erster Linie den aktuellen Verhältnissen zur Last fallen würde. Das geradezu unumstößlich anmutende Dogma des BVerfG von der grundsätzlichen Gleichgewichtigkeit der in die Zukunft hinein zu verteilenden Reduzierungslasten vermag angesichts dessen nicht ohne weiteres zu überzeugen.

Das Gericht ist bei seinen Ausführungen zur eingriffsähnlichen Vorwirkung ersichtlich bemüht, sich nach Möglichkeit weiterhin auf den bewährten Pfaden der traditionellen abwehrcharakterlichen Grundrechts- bzw. Eingriffsdogmatik zu bewegen; vermag aber gerade deshalb die von ihm mit der Figur der ,eingriffsähnlichen Vorwirkung“ selbst geschaffene Ungereimtheit nicht ohne Weiteres auszuräumen. Wird doch bereits mit dem Begriff der „Vorwirkung“ zum Ausdruck gebracht, dass es zur schutzauslösenden Wirkung der Grundrechte des nach allgemein herrschendem Verständnis zu fordernden ,echten“ staatlichen Eingriffs in den Schutzbereich einzelner Grundrechte nicht (mehr) bedürfe, sondern es ausreichen solle, dass ein solcher Eingriff in der Zukunft erwartbar ist bzw. sein kann. Wenn das Gericht wiederum in Anbetracht dessen dieser Vorwirkung Eingriffsähnlichkeit attestiert, orientiert es sich dabei erkennbar immerhin weiter an dem rechtswidrigen staatlichen Eingriff als Voraussetzung für die Verletzung eines Grundrechts; was wiederum die Frage aufwirft, was denn nun mit „eingriffsähnlich“ gemeint sein soll? Nicht etwa doch „Eingriff“? Dafür hätte es aber der auf bloße Ähn-

85) Vgl. auch BVerfG, Beschl. v. 24.3.2021 - 1 BvR 2656/18, 1 BvR 78/20, 1 BvR 96/20, 1 BvR 288/20, Rdnr. 183.

86) BVerfG, Beschl. v. 24.3.2021 - 1 BvR 2656/18, 1 BvR 78/20, 1 BvR 96/20, 1 BvR 288/20, Rdnr. 192. 
lichkeit abstellenden neuen Begrifflichkeit nicht bedurft! Dennoch intendiert das Gericht wohl letztlich genau dies; ,eingriffsähnlich“ soll als eingriffsgleich oder präziser mit einem klassischen Eingriff gleichzusetzen sein oder doch zumindest so behandelt werden. Auf diese Weise erfährt der Eingriffsbegriff also eine Ausweitung. Grundrechtsdogmatisch mag die in der Literatur ${ }^{87}$ überwiegend positiv aufgenommene verfassungsgerichtliche Konstruktion kreativ und innovativ anmuten. Doch darf hierbei nicht außer Acht gelassen werden, dass diese regelmäßig zu Lasten der jetzigen Grundrechtsausübung gehen wird. Aktuelle Freiheitseinschränkungen von Seiten des Staates um der Gewährleistung künftiger Freiheiten willen sind jedoch nicht unproblematisch. Öffnen sie doch fortan die Tür für eine Vielzahl von rechtfertigbaren neuen Grundrechtseingriffen. Man mag sich angesichts dessen fragen, ob die im GG angelegten Grundrechte tatsächlich das richtige Instrument sind, um zumeist vage, auf die Zukunft gerichtete politisch motivierte Schutzmaßnahmen und -erfordernisse durchzusetzen? Alles in allem sollte dem Konstrukt der eingriffsähnlichen Vorwirkung jedenfalls mit einiger Vorsicht begegnet werden.

\subsubsection{Verfassungsrechtliche Rechtfertigung der eingriffsähnlichen Vorwirkung}

Als mögliche verfassungsrechtliche Rechtfertigung für die von $\$ 3$ Abs. 1 S. 2 und $₫ 4$ Abs. 1 S. 3 KSG i.V.m. Anlage 2 ausgehende Gefahr künftiger Freiheitseinbußen (oben 4.2.2.1) rekurriert das Gericht zunächst auf Art. 20a GG. ${ }^{88}$ Mit diesem müssten die erwähnten KSG-Regelungen vereinbar sein. Dies sei nicht der Fall, ,wenn die Vorschriften gegen Art. 20a GG verstießen, weil der verfassungsrechtlich gebotene Klimaschutz nach den dort bis 2030 zugelassenen Emissionsmengen nach 2030 nicht mehr realisiert werden könnte". Auch die Gefährdung künftiger Freiheit durch die genannten KSG-Vorschriften wäre demnach verfassungsrechtlich nicht zu rechtfertigen, wenn diese Vorschriften gegen das Klimaschutzgebot des Art. 20a GG verstießen. ${ }^{89}$ Dies verneint das Gericht, jedoch nicht ohne sich dabei ausführlich mit dieser Staatszielbestimmung auseinanderzusetzen. ${ }^{90}$

Die $\$ 3$ Abs. 1 S. 2 und $\ 4$ Abs. 1 S. 3 KSG i. V.m. Anlage 2 seien ,jedoch insoweit verfassungswidrig, als sie die derzeit nicht hinreichend eingedämmte Gefahr schwerwiegender Grundrechtsbeeinträchtigungen in der Zukunft begründen". ${ }^{91}$ Trotz der ohnehin zweifelhaften Rolle des $\mathrm{CO}_{2}$-Restbudgets (oben 3) verlegt sich das Gericht hier auf die Annahme, dass das noch kleiner sein könnte, als vom SRU eingeschätzt. Unter diesen Umständen müsse der Gesetzgeber sowohl wegen der allgemeinen Verpflichtung schonenden Umgangs mit den Grundrechten, als auch wegen der Verpflichtung, die Gefahr erheblicher Grundrechtsverletzungen einzudämmen, Vorkehrungen zur grundrechtschonenden Bewältigung der nach 2030 drohenden Reduktionslast treffen. ${ }^{92}$ Dies verlange zur Schonung künftiger Freiheit, ,,den Übergang zur Klimaneutralität rechtzeitig einzuleiten“"93. In allen gesellschaftlich relevanten Lebensbereichen müssten Entwicklungen einsetzen, die ermöglichen, dass von grundrechtlicher Freiheit auch später noch dann auf der Grundlage $\mathrm{CO}_{2}$-freier Verhaltensalternativen gehaltvoll Gebrauch gemacht werden könne. ${ }^{94}$ Dies geschieht vor dem Hintergrund, dass - wie das Gericht selbst konstatiert - der Staat schwerlich allein in der Lage sein werde, ,alle technologischen und sozialen Entwicklungen zur Ersetzung und Vermeidung von treibhausgasintensiven Prozessen und Produkten und den Ausbau hierfür erforderlicher Infrastrukturen selbst zu erbringen “95. Dem ungeachtet solle der Staat verfassungsrechtlich verpflichtet sein, grundlegende Vorrausetzungen und Anreize dafür zu schaffen, dass diese Entwicklungen einsetzen.

Inwieweit angesichts dessen am Ende tatsächlich dem Staat die Erreichung der $\mathrm{CO}_{2}-\mathrm{Neutralität}$ auferlegt werden kann, wird so nicht ohne weiteres ersichtlich. Zwar müsse der Staat für effektiven Klimaschutz sorgen. Doch auch er vermag sich nur im Rahmen seiner Möglichkeiten zu bemühen. Nicht zuletzt deswegen stünden - so das BVerfG einmal mehr dem Gesetzgeber Gestaltungsspielräume offen, zumal das GG nicht vorgebe, wie die Voraussetzungen und Anreize für die Entwicklung klimaneutraler Alternativen auszusehen hätten. Gleichwohl sei grundlegend und im Interesse „eine(r) vorausschauende(n) Schonung künftiger Freiheit" zentral, „,dass der Gesetzgeber einer möglichst frühzeitigen Einleitung der erforderlichen Entwicklungs- und Umsetzungsprozesse auch für die Zeit nach 2030 Orientierung bietet und diesen damit zugleich ein hinreichendes Maß an Entwicklungsdruck und Planungssicherheit vermittelt" ${ }^{* 96}$. Dies klingt zunächst wieder einmal durchaus einleuchtend. Allerdings lässt das Gericht Ausführungen zu dem Umstand, dass durch diesen „möglichst frühzeitigen“ Anpassungsdruck die Grundrechtseinschränkungen hier und jetzt erheblich zunehmen, vermissen. Welche grundrechtsrelevanten Dimensionen das Gericht bei den einzuleitenden Maßnahmen im Auge hat, verdeutlicht es immerhin, wenn es davon ausgeht, dass Produkte, Dienstleistungen, Infrastruktur-, Verwaltungs-, Kultureinrichtungen sowie Konsumgewohnheiten erheblich umzugestalten seien"97. Gerade, wenn diese Maßnahmen vorzeitige Erfolge zeitigten, wäre aber aus der expost-Perspektive die aktuelle Belastung der Grundrechtsträger wohl ungleich höher als die derjenigen, zu deren Gunsten diese Maßnahmen in der Zukunft erfolgten.

\section{Verordnungsermächtigung des $\$ 4$ Abs. 6 S. 1 KSG i.d.F.v. 2019}

Ist es schon schwierig die Zeitspanne bis 2030 in ihrer Entwicklung seriös vorherzusehen, gilt dies erst recht für den Zeitraum danach bis 2050. Das BVerfG beanstandet in Anbetracht dessen auch nicht, den in den KSG-Vorschriften angelegten $\mathrm{CO}_{2}$-Reduzierungsplan bis 2030 (oben 4.2.2.2), sondern nur die Reduzierungsanstrengungen für die Zeit danach, die laut $\$ 4$ Abs. 6 S. 1 KSG i.d. F.v. 2019 einer im Jahr $2025 \mathrm{zu}$ erlassenden Verordnungsregelung vorbehalten bleiben sollen. ${ }^{98}$ Es moniert insoweit, dass die konkrete Fortschreibung in $\$ 4$ Abs. 6 S. 1 KSG unzureichend geregelt sei, denn sie genüge den verfassungsrechtlichen Anforderungen an eine der weiteren Entwicklung hinreichende Orientierung vermittelnde Ausgestaltung im Ergebnis nicht. ${ }^{99}$ Insbesondere sei ein wiederholter Festlegungsprozess auch unter dem Gesichtspunkt zeitlich hinreichend

87) Vgl. etwa Schlacke, NVwZ 2021, 912, $913 \mathrm{f}$.

88) BVerfG, Beschl. v. 24.3.2021 - 1 BvR 2656/18, 1 BvR 78/20, 1 BvR 96/20, 1 BvR 288/20, Rdnr. $190 \mathrm{ff}$.

89) BVerfG, Beschl. v. 24.3.2021 - 1 BvR 2656/18, 1 BvR 78/20, 1 BvR 96/20, 1 BvR 288/20, Rdnr. 190.

90) BVerfG, Beschl. v. 24.3.2021 - 1 BvR 2656/18, 1 BvR 78/20, 1 BvR 96/20, 1 BvR 288/20, Rdnr. $196 \mathrm{ff}$.

91) BVerfG, Beschl. v. 24.3.2021 - 1 BvR 2656/18, 1 BvR 78/20, 1 BvR 96/20, 1 BvR 288/20, Rdnr. 195, 243.

92) BVerfG, Beschl. v. 24.3.2021 - 1 BvR 2656/18, 1 BvR 78/20, 1 BvR 96/20, 1 BvR 288/20, Rdnr. 247.

93) BVerfG, Beschl. v. 24.3.2021 - 1 BvR 2656/18, 1 BvR 78/20, 1 BvR 96/20, 1 BvR 288/20, Rdnr. 248.

94) BVerfG, Beschl. v. 24.3.2021 - 1 BvR 2656/18, 1 BvR 78/20, 1 BvR 96/20, 1 BvR 288/20, Rdnr. 248.

95) BVerfG, Beschl. v. 24.3.2021 - 1 BvR 2656/18, 1 BvR 78/20, 1 BvR 96/20, 1 BvR 288/20, Rdnr. 248.

96) BVerfG, Beschl. v. 24.3.2021 - 1 BvR 2656/18, 1 BvR 78/20, 1 BvR 96/20, 1 BvR 288/20, Rdnr. 249.

97) BVerfG, Beschl. v. 24.3.2021 - 1 BvR 2656/18, 1 BvR 78/20, 1 BvR 96/20, 1 BvR 288/20, Rdnr. 249.

98) BVerfG, Beschl. v. 24.3.2021 - 1 BvR 2656/18, 1 BvR 78/20, $1 \mathrm{BvR}$ 96/20, 1 BvR 288/20, Rdnr. $256 \mathrm{ff}$.

99) BVerfG, Beschl. v. 24.3.2021 - 1 BvR 2656/18, 1 BvR 78/20, 1 BvR 96/20, 1 BvR 288/20, Rdnr. 256. 
weitgreifender Festlegung unerlässlich. ${ }^{100}$ Zwar könne nich verlangt werden, dass die abzusenkenden $\mathrm{CO}_{2}$-Emissionsmengen jetzt bis zum Ende, also bis zur Erreichung der für 2050 angestrebten Klimaneutralität konkret bestimmt würden. Doch genüge es nicht, dass $\$ 4$ Abs. 6 S. 1 KSG die Bundesregierung lediglich dazu verpflichte, im Jahr 2025 für weitere Zeiträume nach dem Jahr 2030 jährlich sinkende Emissionsmengen durch Rechtsverordnung festzulegen. Damit bliebe nämlich offen, wieweit in die Zukunft diese Festlegung reichte. Nach dem Wortlaut der Verordnungsermächtigung - so das Gericht - könnte es sich dabei auch um bloß zwei Ein-Jahres-Zeiträume handeln, die lediglich bis 2031 reichten. Gerade weil der Reduzierungspfad im Jahr 2025 kaum endgültig festgelegt werden könne und solle, genüge es nicht, die Bundesregierung lediglich dazu zu verpflichten, im Jahr 2025 einmal eine weitere Festlegung zu treffen. Es müsse zumindest bestimmt werden, in welchen Zeitabständen weitere Festlegungen transparent $\mathrm{zu}$ treffen seien. ${ }^{101}$ Außerdem wird moniert, dass bis 2025 keine Planung über das Jahr 2030 hinaus bestehe, weswegen es zweifelhaft erschiene, dass die erste weitere Festlegung von $\mathrm{CO}_{2}$-Jahresemissionsmengen in Zeiträumen nach 2030 rechtzeitig käme. Vornehmlich fürchtet das Gericht, dass in vielen Produktions- und Konsumbereichen ein Vorlauf von bloß 5 Jahren nicht genügen würde, die zur späteren Schonung der Grundrechte erforderliche Entwicklung rechtzeitig anzustoßen. ${ }^{102}$ Es erachtet zeitlich hinreichend weitgreifendere Festlegungen für unerlässlich. Müssten doch die Rahmenbedingungen für die diversen Produktions- und Konsumbereiche möglichst frühzeitig steuernd umrissen werden, um rechtzeitig reduzierungsrelevante Maßnahmen für die einzelnen Reduzierungspfade ergreifen zu können.

Sofern indes das BVerfG mit Blick auf das Klimaschutzgebot des Art. 20a GG der Ansicht ist, dass Art. 20a GG die Festlegung von $\mathrm{CO}_{2}$-Jahresemissionsmengen fordere, ${ }^{103}$ wird es wohl dessen Geheimnis bleiben, woraus sich dies im Art. 20a GG ergeben sollte. Allein der Umstand, dass in $\$ 3$ Abs. 1 S. 2 und $\$ 4$ Abs. 1 S. 3 KSG i. V.m. Anhang 2 bis zum Jahr 2030 tatsächlich Jahresemissionsmengen festgesetzt sind, darf jedenfalls nicht damit gleichgesetzt werden, dass dies aus Art. 20a GG selbst resultiere (vgl. auch oben 2); erst recht kann daraus für den Gesetzgeber kein Automatismus für den Zeitraum danach abgeleitet werden. Die dem Gesetzgeber aufgegebene Jahresemissionsmengenregelungspflicht für die Zeit nach 2030 überzeugt mit dieser Begründung daher keineswegs. Denkbar wären mit Blick auf Art. 20a GG nämlich auch etwa größere zeitliche Intervalle; selbst eine Festlegung ohne bestimmte Emissionsmengenreduzierungen widersprächen den vagen Vorgaben des Klimaschutzgebots aus Art. 20a GG grundsätzlich nicht. Art. 20a GG schreibt mitnichten einen bestimmten Reduzierungsweg vor. Die Reduzierung hat lediglich zielführend zu sein, das heißt, das insoweit in Konkretisierung dieser Staatszielbestimmung gesteckte Ziel $\left(\mathrm{CO}_{2}-\mathrm{Neutrali}-\right.$ tät bis 2050) muss erreicht werden. Anstatt der eigenen Jahresemissionsmengenregelung - so das Gericht - könne der Gesetzgeber aber auch dem Verordnungsgeber wesentliche Kriterien für die Bemessung der jährlichen Mengen vorgeben. Denkbar sei etwa, dass der Gesetzgeber Minderungsquoten für bestimmte Zieljahre vorgebe. Weil diese Quoten „,für sich genommen nicht aussagekräftig“ seien, müsste er dann aber zusätzlich nähere Vorgaben zu dem zum Zieljahr führenden Reduzierungspfad machen. ${ }^{104}$ Auch hier vermögen die Ausführungen des Gerichts nicht zu überzeugen, da ebenso wie die Herleitung von Jahresemissionsmengen auch diejenige bestimmter Minderungsquoten aus Art. 20a GG schwerlich zwingend ist. Dabei hätte sich das Gericht bei Berücksichtigung des jedenfalls auch im Rahmen verfassungsrechtlicher Erörterungen maßgeblichen einschlägigen EU-Rechts die Argumentation erheblich einfacher machen können. Verpflichtet doch etwa Art. 3 i.V.m. Art. 4 lit. a) Nr. 1 lit. i) der Verordnung (EU) 2018/1999 ${ }^{105}$ i. V.m. der Verordnung (EU) 2018/842 ${ }^{106}$ die Mitgliedstaaten bereits auf jährliche verbindlich festzusetzende nationale Obergrenzen für Treibhausgasemissionen.

Die vom BVerfG gewählte Argumentation erscheint indes als eine Anmaßung, wenn es (ohne Berücksichtigung EUrechtlicher Vorgaben) dem Gesetz- wie auch dem Verordnungsgeber derartige Reduzierungsvorgaben macht. Konsequenterweise müsste nämlich die Wahl der Mittel - was das Gericht in anderem Zusammenhang nicht müde wird, $\mathrm{zu}$ betonen - zumindest dem demokratisch legitimierten Gesetzgeber als Forum gesellschaftsrelevanter Diskussionen selbst überlassen bleiben. Eine nicht einmal in Art. 20a GG ausdrücklich aufgeführte staatliche Pflicht zum Klimaschutz vermag derart weitgehende Ableitungen schwerlich zu rechtfertigen. Ungeachtet dessen setzt sich das Gericht an die Stelle des Gesetzgebers; zumindest aber zeigt es diesem die von ihm „,gefälligst“ einzuschlagenden Wege auf. Gerade wenn ein Diskurs im Parlament stattfindet bzw. stattgefunden hat, gebietet es das Demokratieprinzip, etwaig getroffene inhaltliche Entscheidungen zu respektieren und nicht an deren Stelle die eigenen Vorstellungen des Gerichts zu setzen. Auch wenn das Gericht an dieser Stelle die politische Entwicklung im Bereich des Klimaschutzes zu befördern gedenkt, so sei daran erinnert, dass dies nicht dessen originäre Aufgabe ist. Es hat einzig darüber zu wachen, dass Maßnahmen der anderen Verfassungsorgane mit dem geltenden Verfassungsrecht im Einklang stehen.

\section{Gesetzgeberischer Anpassungsbedarf}

Für den Gesetzgeber folgt hieraus, dass er bis zum 31.12.2022 die Fortschreibung der Minderungsziele für Zeiträume nach 2030 unter Beachtung der Maßgaben der hiesigen Entscheidung näher zu regeln hat. ${ }^{107} \mathrm{D}$. h. es muss zumindest eine mit wesentlichen Eckpunkten versehene Langfriststrategie bis zur Erreichung der Klimaneutralität entwickelt werden. ${ }^{108}$ Diesem Auftrag ist der Gesetzgeber mittlerweile durch eine entsprechende Anpassung des $\mathrm{KSG}^{109}$ nachgekommen. ${ }^{110}$

100) BVerfG, Beschl. v. 24.3.2021 - 1 BvR 2656/18, 1 BvR 78/20, 1 BvR 96/20, 1 BvR 288/20, Rdnr. 258.

101) BVerfG, Beschl. v. 24.3.2021 - 1 BvR 2656/18, 1 BvR 78/20, 1 BvR 96/20, 1 BvR 288/20, Rdnr. 257.

102) BVerfG, Beschl. v. 24.3.2021 - 1 BvR 2656/18, 1 BvR 78/20, 1 BvR 96/20, 1 BvR 288/20, Rdnr. 258.

103) BVerfG, Beschl. v. 24.3.2021 - 1 BvR 2656/18, 1 BvR 78/20, 1 BvR 96/20, 1 BvR 288/20, Rdnr. 263: „Anderes ließe Art. 20a GG auch nicht zu“.

104) BVerfG, Beschl. v. 24.3.2021 - 1 BvR 2656/18, 1 BvR 78/20, $1 \mathrm{BvR}$ 96/20, 1 BvR 288/20, Rdnr. 264

105) Verordnung (EU) 2018/1999 des Europäischen Parlament und des Rates über das Governance-System für die Energieunion und für den Klimaschutz, zur Änderung der Verordnungen (EG) Nr. 663/2009 und (EG) Nr. 715/2009 des Europäischen Parlaments und des Rates, der Richtlinien 94/22/EG, 98/70/EG, 2009/31/EG, 2009/73,/EG, 2010/31/EU, 2012/27/EU und 2013/30/EU des Europäischen Parlaments und des Rates, der Richtlinien 2009/119/EG und (EU) 2015/652 des Rates und zur Aufhebung der Verordnung (EU) Nr. 525/2013 des Europäischen Parlaments und des Rates vom 11.12.2018, ABl. 2018 L 328, S. 1.

106) Verordnung (EU) 2018/842 des Europäischen Parlament und des Rates zur Festlegung verbindlicher nationaler Jahresziele für die Reduzierung der Treibhausgasemissionen im Zeitraum 2021 bis 2030 als Beitrag zu Klimaschutzmaßnahmen zwecks Erfüllung der Verpflichtungen aus dem Übereinkommen von Paris sowie zur Änderung der Verordnung (EU) Nr. 525/2013 vom 30.5.2018, ABl. 2018 L 156, S. 26.

107) BVerfG, Beschl. v. 24.3.2021 - 1 BvR 2656/18, 1 BvR 78/20, 1 BvR 96/20, 1 BvR 288/20, Rdnr. 268.

108) Schlacke, NVwZ 2021, 912, 916.

109) Erstes Gesetz zur Änderung des Bundes-Klimaschutzgesetzes v. 18.8.2021, BGBl. I S. 3905.

110) Näher dazu etwa Jope, EWeRK 2021, $145 \mathrm{ff}$. 


\section{Mögliche Implikationen des Beschlusses}

Bei seinen überaus ausführlichen Darstellungen und den den gesamten Beschluss durchziehenden Duktus, dass die $\mathrm{CO}_{2}$ Reduzierung mit Recht eine aus Art. 20a GG abgeleitete Verpflichtung des Staates sei, lässt das ersichtlich für grundrechtsrelevante $\mathrm{CO}_{2}$-Reduzierungsmaßnahmen aufgeschlossene BVerfG offen, inwieweit der Staat sich objektiv wirksamen Maßnahmen enthalten kann und darf. Würden doch bereits bestehende, aber nicht oder nur unzureichend genutzte Möglichkeiten zur $\mathrm{CO}_{2}$-Vermeidung jedenfalls einen Beitrag $\mathrm{zu}$ weniger grundrechtsrelevanten Beschränkungen bedeuten. Angesichts dessen wäre es nämlich unter Umständen sogar eine Frage der Verhältnismäßigkeit, ob auf Technologien - wie die , $\mathrm{CO}_{2}$-neutrale“ Kernenergie - zur Erreichung des „Paris-Ziels“ einfach verzichtet werden darf. Müsste nicht der alsbald anstehende endgültige „Ausstieg“ Deutschlands aus der Stromerzeugung mittels Kernenergie ausgesetzt werden und im Mindesten die derzeit noch betriebenen Atommeiler zur Stromversorgung weiterlaufen; bestünde nicht vielleicht sogar angesichts der derzeitigen Energieversorgungssituation sogar die Pflicht zum Neubau neuer Kernkraftwerke? Pointierter gefragt: Verletzt der Staat nicht etwa seine aus Art. 2 Abs. 2 S. 1 GG resultierende Schutzpflicht, wenn er auf die aktuell nutzbare Kernkraft verzichtet?

Auch stellt sich angesichts der vom BVerfG entwickelten intertemporalen Freiheitssicherung die Frage, inwieweit die Möglichkeit zur künftigen Grundrechtsausübung zum Maßstab für die Einschränkungen jetziger Freiheiten auch in anderen Bereichen werden kann. So könnte gerade mit Blick auf die ebenfalls in Art. 20a GG verankerte staatliche Pflicht zum Umweltschutz vergleichbares gelten. Wäre es wohl nicht ausgeschlossen, dass etwa im Falle einer für Deutschland in $\mathrm{Zu}-$ kunft für den Wasserhaushalt prognostizierten Wasserknappheit grundrechtlich relevante Einschränkungen beim privaten Wasserverbrauch (vorsorglich) schon jetzt vorgenommen werden. In konsequenter Fortschreibung der richterlichen Vorgehensweise könnte unter Umständen sogar eine Budgetierung aller derzeit vom Menschen stark oder übermäßig in Anspruch genommenen natürlichen Lebensgrundlagen in Betracht kommen, um nur in Zukunft der jüngeren Generation erhebliche(re) Einschränkungen zu ersparen.

\section{Fazit}

Das BVerfG will durch Freiheitseinschränkungen Freiheit sichern. Es unterstellt etwas vereinfacht, dass im Wesentlichen nur Verbote und staatliche Lenkungsmaßnahmen für den Klimaschutz zielführend sein könnten. Dies unterschätzt aber zumindest die Dynamik der wissenschaftlichen und gesellschaftlichen Fortentwicklung in einem freiheitlichen und marktwirtschaftlich ausgerichteten modernen Industrieland.

Ein Aspekt auf den das Gericht im Übrigen überhaupt nicht eingeht, ist die Frage, ob das in \$1 S. 3 KSG zugrundegelegte „Paris-Ziel“ für Deutschland überhaupt erreichbar scheint. Es wird einfach als zwingend zu erreichend vorausgesetzt. Dass sich Deutschland international zur Erreichung der Pariser Klimaschutzziele verpflichtet hat und \$1 S. 3 KSG diese Ziele folgerichtig übernimmt, mag zwar zunächst durchaus eine zulässige und sinnvolle Konkretisierung des Klimaschutzgebotes in Art. 20a GG darstellen, ist aber keine sich unmittelbar aus Art. 20a GG ergebende Obliegenheit. Vielmehr muss auch dieses Temperaturbegrenzungsziel zunächst an Art. 20a GG selbst gemessen werden. Gerade die Frage einer realistischen Erreichbarkeit dieser Ziele muss dabei im Vordergrund stehen. Dass aber überhaupt keine Zweifel an deren tatsächlicher Erreichbarkeit geäußert wird, bzw. diese nicht ausgeräumt oder zumindest angesprochen werden, wirkt befremdlich. Ist dies doch angesichts der mit den $\mathrm{CO}_{2}$-Begrenzungszielen teilweise verbundenen gravierenden grundrechtsrelevanten Eingriffsmaßnahmen von zentraler Bedeutung. Vor diesem
Hintergrund vermag nicht zuletzt auch die vom Gericht aus den Grundrechten hergeleitete ,eingriffsähnliche Vorwirkung" in einem anderen Licht zu erscheinen.

Auch lässt das BVerfG weitgehend offen, inwieweit sich die Grundrechte der Erreichung eines solchen Zieles jeweils zu unterwerfen hätten. Ein in einer freiheitlichen Gesellschaft aber essentieller Aspekt. Der Klimaschutz ist zwar zu Recht ein Wert von Verfassungsrang, er darf aber nicht zu einem Instrument der Grundrechtseliminierung degenerieren. Dies gebietet letztendlich das Verhältnis des Art. 20a GG zu den Grundrechten. Denn in beiden Fällen handelte es sich um Werte von Verfassungsrang, deren Kollision nur im Wege der praktischen Konkordanz zu lösen wäre. Was nichts anderes bedeutet, als das jeder der betroffenen Verfassungsgüter im Lichte der Verfassung zur größtmöglichen Bedeutung gebracht werden müsste. Ein Wegwägen von Grundrechtspositionen wäre jedenfalls unzulässig. Über weite Strecken erweckt die Entscheidung des BVerfG aber eher den Eindruck, als hätten sich die Grundrechte den Belangen des Klimaschutzes unterzuordnen. Nur so lässt sich erklären, dass das Gericht zur Erreichung der nicht ernsthaft hinterfragten Klimaschutzziele höheren grundrechtsrelevanten Belastungen in der Zukunft entgegenwirken möchte und sich deshalb auch bereit zeigt, gegebenenfalls den Grundrechtsträgern hier und jetzt weitere Beschränkungen aufzuerlegen. Im Übrigen geht das Gericht auf den Aspekt einer möglichen Vereinbarkeit der deutschen Klimaschutzziele mit dem EU-Recht viel zu oberflächlich ein. ${ }^{111}$

Ungeachtet dessen fallen die sehr umfänglichen Einlassungen des BVerfG mitunter einfach nur unübersichtlich und teilweise unnötig redundant aus. Sie erwecken - wie etwa diejenigen über das Restbudget - den Eindruck einer nicht immer stringenten Gedankenführung, die auch vor einer Reihe von Widersprüchlichkeiten nicht Halt macht. Das Gericht verliert sich - nicht zuletzt bei seiner Würdigung des aktuellen Standes der „Klima“-Wissenschaft - des Öfteren in seitenlangen Ausführungen, die eigentlich mit den das Ergebnis des Beschlusses tragenden Gründen nur bedingt etwas zu tun haben.

Der Beschluss des BVerfG zeigt einmal mehr, dass politisch Notwendiges und Wünschbares eben auch von der Politik umgesetzt werden muss. Das Verfassungsrecht und mehr noch das BVerfG vermag das demokratisch legitimierte politische Handeln nicht zu ersetzen. Das Gericht stößt ersichtlich an seine Grenzen, wenn es - wie hier dem GG konkrete Maßgaben für eigentlich politisch motivierte Ziele abringen möchte. Die allenthalben zunehmenden Versuche der „Weltrettung per Gerichtsbeschluss“112 dürften daher ein Irrweg sein.

Open Access. Dieser Artikel wird unter der Creative Commons Namensnennung 4.0 International Lizenz veröffentlicht, welche die Nutzung, Vervielfältigung, Bearbeitung, Verbreitung und Wiedergabe in jeglichem Medium und Format erlaubt, sofern Sie den/die ursprünglichen Autor(en) und die Quelle ordnungsgemäß nennen, einen Link zur Creative Commons Lizenz beifügen und angeben, ob Änderungen vorgenommen wurden.

Die in diesem Artikel enthaltenen Bilder und sonstiges Drittmaterial unterliegen ebenfalls der genannten Creative Commons Lizenz, sofern sich aus der Abbildungslegende nichts anderes ergibt. Sofern das betreffende Material nicht unter der genannten Creative Commons Lizenz steht und die betreffende Handlung nicht nach gesetzlichen Vorschriften erlaubt ist, ist für die oben aufgeführten Weiterverwendungen des Materials die Einwilligung des jeweiligen Rechteinhabers einzuholen.

Weitere Details zur Lizenz entnehmen Sie bitte der Lizenzinformation auf http://creativecommons.org/licenses/by/4.0/deed.de.

Open Access funding enabled and organized by Projekt DEAL.

111) Vgl. BVerfG, Beschl. v. 24.3.2021 - 1 BvR 2656/18, 1 BvR 78/20, 1 BvR 96/20, 1 BvR 288/20, Rdnr. 141.

112) Faßbender, NJW 2021, 2085, 2088 